\title{
Combination rule for redundant information in reaction time tasks with divided attention
}

\author{
G. ROBERT GRICE, LYN CANHAM, and JOSEPH M. BOROUGHS \\ University of New Mexico, Albuquerque, New Mexico
}

\begin{abstract}
Five choice reaction time experiments were conducted to investigate the redundant-signals effect in letter identification. In two experiments, displays were large letters formed by small letters. Three experiments used simple two-letter displays with different spatial arrangements. Data were analyzed by the measurement model of variable criterion theory. In all experiments, throughout the RT distributions, the strength of the redundant signals condition was an extremely precise linear function of the sum of the strengths of the separate components. Each stimulus component contributed about one-half of its strength. The form of the distribution was entirely determined by this additive relation. However, the superiority of the redundant-signals condition is not produced by this summation, but by an additive constant present throughout the distribution. It is suggested that this represents a decrement produced by a distractor in a relevant location in single-target conditions. The results strongly favor strength theory as opposed to separate activation models that account for the redundant-signals effect by statistical facilitation. Additional information provided concerns the global precedence effect and S-R compatibility.
\end{abstract}

When human observers monitor two sources of information for a target, performance is facilitated if the target is present in both sources rather than only one. This finding has been called the redundant-signals or redundant-targets effect (Kinchla, 1974). Miller (1982) has recently distinguished two classes of theoretical accounts of this phenomenon.

In one approach, the observer is conceived as processing the two sources of information separately, but in parallel and not necessarily at the same rate. An affirmative decision is made if the target is detected in either source. If the response measure is reaction time (RT), the decision to respond is made when the target is detected in the first channel. The decision mechanism may be considered an analog of an OR gate. If the detection-time distributions of the two channels overlap, then the average RT will be faster with two signals present than with only a single source. Raab (1962) appropriately called this explanation of the redundant-signals effect "statistical facilitation." Such an account is an example of a "race" model. Miller (1982) used the term "separate activation" for this approach and cited a number of the investigators who have used it. More recent examples are two attention-sharing models, based on independence, presented by Shaw (1982). However, her models were applied to detection experiments only, and she did not suggest their extension to RT data.

The second class of theories described by Miller (1982) is characterized by "coactivation." In this

This research was supported by PHS Grant MH16400 from the National Institute of Mental Health. Requests for reprints should be sent to G. Robert Grice, Department of Psychology, University of New Mexico, Albuquerque, NM 87131. view, "activation" increases gradually following stimulus onset until a criterion is reached. With two inputs, the two sources of information combine and the growth of activation is more rapid. Following the electronic analogy, the mechanism leading to the decision to respond is analogous to an integrator rather than an OR gate. This view is entirely consistent with the continuous flow conception of C. W. Eriksen and Schultz (1979), and with our own variable criterion theory approach (Grice, Canham, \& Schafer, 1982). However, we have not previously analyzed redundant stimulus inputs from the point of view of variable criterion theory. C. W. Eriksen and Schultz regard the growth of activation as dependent on "response priming." In variable criterion theory, the terms used are "excitatory strength" or "associative strength." The present research is concerned with letter identification in visual displays.

Miller $(1981,1982)$ has proposed an interesting and important basis for evaluating the class of separate activation theories. The method is derived from the race property of these models, and is based on the information in the cumulative RT distribution functions for the separate and combined stimulus inputs. He points out that, according to such models, when both stimuli (A and B) are present, the following relation holds for all values of time $(t)$ following stimulus onset:

$$
\begin{aligned}
& P(R T<t \mid A \text { and } B) \\
& =P(R T<t \mid A)+P(R T<t \mid B)-P[(R T<t \mid A) \\
& \text { and }(R T<t \mid B)] .
\end{aligned}
$$


For independent-channels models, the subtractive term is merely the product of the two separate probabilities. If the channels are not independent, the subtractive term becomes

$$
[P(R T<t \mid A)][P(R T<t \mid B) \mid(R T<t \mid A)] .
$$

If the channels are positively correlated, the term will be greater than the product for independence, and if they are negatively correlated, it will be less than the product. In the special case of a perfect negative correlation, the subtractive term will be zero. This sets an upper limit on response probability when both stimuli are present, since

$$
\begin{aligned}
P(R T<t \mid A \text { and } B) \leqslant P(R T<t \mid A) & \\
& +P(R T<t \mid B) .
\end{aligned}
$$

Thus, if, at any time in the cumulative RT distribution functions, the probability of response to the combined stimuli is greater than the sum of the two separate probabilities, Inequality 2 is violated. In this case, this entire class of separate activation models may be rejected for the particular set of data.

From the point of view of separate activation theories, a negative correlation between two channels in divided-attention tasks would not be unexpected. This is because of the possibility that the channels may compete for resources. Even so, it should be recognized that Miller's proposed test is extremely conservative. One would not realistically expect a negative correlation to be perfect. This is particularly true in view of evidence that the cost of dividing attention is quite small unless separate responses are required to the two inputs (e.g., see Duncan, 1980).

Miller has also developed a procedure for applying this analysis to group data. The analysis is applied to average cumulative distributions obtained by the method of Ratcliff (1979). Throughout a cumulative distribution, quantiles are computed at evenly spaced percentages for each subject separately. These quantiles are then averaged over subjects at the corresponding percentages. This produces an average distribution with quantiles at the means of the subject quantiles. Miller obtained a distribution in this way for the condition in which both stimuli were present. He also constructed a second "distribution," which, for all times, consisted of the sums of the two cumulative distributions for separate presentation. The same quantiles were obtained for this distribution. If, at any time, a quantile is reached earlier by the "both" distribution than by the distribution of sums, Inequality 2 is violated. The statistical significance of such a violation is evaluated by means of a t test. Since the distribution of sums approaches two rather than one, meaningful comparisons may be made only at relatively early quantiles.
Miller (1982) applied this analysis to three bimodal detection experiments, finding significant violations of Inequality 2. However, the present research is not directed to that problem. He also presented three experiments involving the visual detection of letter targets. One of these (Miller, 1981) was conducted in the context of global precedence (Navon, 1977). The stimuli were large letters made up of small ones, and the target could appear at the global or local level or at both. The other experiments (Miller, 1982) involved two letters separated horizontally, the target appearing in either of the two positions or in both. A position or level not containing a target was filled by a neutral letter. There were trials with no target, and the subject responded by pressing a "yes" or a "no" key. In each of the experiments, Inequality 2 was significantly violated in the early portions of the distributions. Miller concluded that the class of separate activation models must be rejected, and that a coactivation model with a summation principle was required.

In spite of the consistency and apparent clarity of Miller's results, we believe that there is a possible flaw in the experiments that could potentially negate the conclusions. The difficulty is that there can be no truly neutral stimulus in this situation. Any letter other than a target may tend to evoke the "no" response, producing the kind of associative interference discovered by B. A. Eriksen and C. W. Eriksen (1974) and since studied by a number of investigators (e.g., C. W. Eriksen \& Shultz, 1979; Grice, Canham, \& Shafer, 1982; Flowers \& Wilcox, 1982). Thus, the difference between single- and double-target trials may be due, at least in part, to interference that accompanies a single target but is absent when two targets are present.

Our first purpose here is to use Miller's rationale for evaluating separate activation theory while avoiding the difficulty inherent in the yes-no design. We have used a choice reaction time (CRT) procedure with two target letters, one mapped on each of two responses. In this situation, a nontarget letter is more truly neutral, in that it is not associated with either response. On each trial, one of the two targets is presented, either to both channels or singly and accompanied by an irrelevant letter. Five such experiments are presented. Two involve displays of a large letter made up of small ones. The remaining three use twoletter displays with differing spatial arrangements. Two of them include the factor of S-R compatibility in the analysis.

The other and major aim of the research is to apply the measurement procedures of variable criterion theory to an investigation of the rules by which information combines when two redundant stimulus inputs are present. This analysis is also based on the cumulative RT distributions. From the point of view of variable criterion, strength theory, these functions 
are taken to reflect the growth of associative strength. With three distributions, it is possible to examine the growth of associative strength for each stimulus separately and for the two in combination when both are present. The five experiments provide considerable variety in the relations between component functions. If a common combination rule should apply to all of the experiments, considerable generality would be indicated.

\section{EXPERIMENTS 1 AND 2}

In the first two experiments, the stimuli were the large letters made up of small ones that were used by Grice, Canham, and Boroughs (1983) in investigating global precedence. This is the class of stimuli used in the first Miller (1981) experiment. The target letter was presented either at the global or local level or at both. In Experiment 1, the stimulus appeared irregularly to the right or left of the fixation point, a condition that produced the global precedence effect in the earlier research. In Experiment 2, the stimulus was always centered at the fixation point. This condition was found not to produce the global precedence effect. For separate presentation and uncertain stimulus location, we should expect faster response to the large target than to the small one. With fixed location, we should expect RTs to be about the same to the two kinds of target when presented separately. The interest is in whether the manipulation will affect the redundant signals effect or the way in which it is produced.

\section{Method}

Stimulus displays and general procedures were as described by Grice et al. (1983). The stimuli, presented on a video monitor by a TRS-80 computer, were large capital letters made up of small capital letters. The small letters were formed by dots in a $5 \times 7$ matrix and were approximately .29 deg wide and .35 deg high. The large letters were formed in the same manner in a $5 \times 7 \mathrm{ma}$ trix with the dots replaced by small letters. The large letters were approximately $2.76 \mathrm{deg}$ wide and $3.57 \mathrm{deg}$ high. The two target letters were $\mathbf{H}$ and $\mathbf{S}$. The subjects were instructed to respond to one of the targets by pressing a key with the right index finger and to the other target by pressing a second key with the left index finger. The two mappings of right and left on the targets were divided equally among the subjects. Instructions were to respond as quickly as possible while avoiding errors. RTs were recorded in milliseconds.
One of the target letters was presented on each trial. Equally often the target was the large letter, the small letter, or both the large and small letters. On trials with a single target, the irrelevant letter was $Y$. This letter was selected from the available font of letters to minimize similarity with both targets. A single irrelevant letter was used, rather than several, to simplify the task and to eliminate any extra processing time that might result from a variety of irrelevant letters. The targets, $H$ and $S$, were presented equally often in each of the three kinds of trials. A plus sign, used as a fixation point, was continuously in the center of the screen except when the stimuli were on.

The warning signal before each trial was a $.5-\mathrm{sec}, 1000-\mathrm{Hz}$ tone of $70 \mathrm{~dB}$ SPL. The subjects were instructed to look at the fixation point at the tone and be prepared to respond. The stimulus came on $1 \mathrm{sec}$ after the onset of the tone. The interval between trials was $4 \mathrm{sec}$. The stimuli remained on the screen until the subject responded. In the event of an error, the word ERROR appeared on the screen for $.5 \mathrm{sec}$ and there was a $.5-\mathrm{sec}$ burst of noise of $70 \mathrm{~dB}$ SPL. The experiment began with 50 practice trials followed by a short rest. There were then 250 trials without interruption. Data were based on the final 240 trials, 80 of each kind.

In Experiment 1, the stimulus appeared irregularly and equally often with its center 2.47 deg to the right or left of the fixation point. In Experiment 2, the stimulus was always centered at the fixation point.

The subjects in each experiment were groups of 28 female undergraduate students from courses in introductory psychology. They received course credit for participation.

\section{Results}

Mean RTs and error rates for each condition in the two experiments are presented in Table 1. The redundant-signals effect was obtained in both experiments and did not differ greatly between them. Probably the best comparison is between presentation at both levels and the faster of the two single-level conditions. In Experiment 1 with uncertain location, this was the large letter, but in Experiment 2, RTs to the small letter were slightly faster. When the comparison is made for the two experiments, the redundantsignals effect is significant $[F(1,54)=163.76, p<$ $.001]$. The interaction, experiments $\times$ single vs. double targets, was not significant $[F(1,54)<1]$. The fixedlocation experiment was significantly faster than the uncertain-location experiment $[F(1,54)=5.56, p<$ $.025]$.

Also of interest is an evaluation of the global precedence effect, which predicts that RTs should be faster to the single large target than to the small target. Such a difference was obtained only in Experiment 1 . When the two experiments are analyzed to-

Table 1

Means and Standard Deviations of Mean Correct Reaction Time (in Milliseconds) and of Error Rates in Experiment 1 (Uncertain Location) and Experiment 2 (Fixed Location)

\begin{tabular}{|c|c|c|c|c|c|c|c|c|}
\hline \multirow{3}{*}{$\begin{array}{c}\text { Target } \\
\text { Condition }\end{array}$} & \multicolumn{4}{|c|}{ Reaction Time } & \multicolumn{4}{|c|}{ Error Rate } \\
\hline & \multicolumn{2}{|c|}{ Uncertain Location } & \multicolumn{2}{|c|}{ Fixed Location } & \multicolumn{2}{|c|}{ Uncertain Location } & \multicolumn{2}{|c|}{ Fixed Location } \\
\hline & Mean & SD & Mean & SD & Mean & SD & Mean & SD \\
\hline Both & 512 & 84 & 457 & 67 & .017 & .018 & .016 & .016 \\
\hline Large & 573 & 107 & 529 & 73 & .013 & .013 & .021 & .023 \\
\hline Small & 624 & 97 & 525 & 68 & .036 & .033 & .026 & .034 \\
\hline
\end{tabular}


gether, RT to the large target is significantly faster, due entirely to the uncertain location experiment $[F(1,54)=12.60, p<.001]$. The interaction, target size $\times$ certainty condition, was also significant $[F(1,54)$ $=17.75, \mathrm{p}<.001]$. This confirms the finding of Grice et al. (1983), in focused attention experiments, that global precedence is found with uncertain location but not with fixed, central location of the stimulus.

Error rates, as expected, were generally low. The only significant effect was in Experiment 1 , in which the error rate was greater for the small target than for the other two conditions. By the sign test, $p<.01$ for both comparisons.

Tests of separate activation theory. In applying Miller's $(1981,1982)$ procedures to the cumulative distribution functions, we are interested only in correctresponse distributions. Ideally, the distributions should be error-free. In spite of the low error rates here, they should be corrected for the presence of errors. There are several ways in which this might be done. However, we believe that the best method is applying the equations of Grice, Spiker, and Nullmeyer (1979) for their race model for correct responses and errors. This procedure yields correctresponse distributions that are invariant in form with variations in error rate. In other words, the distribution is what it would be without errors. This property of the procedure has been confirmed both by simulation and empirically in research with experimental manipulation of the speed-accuracy tradeoff. The equations have more recently been presented in an appendix by Grice, Canham, and Schafer (1982). All distributions analyzed and presented in this article are based on corrected distributions of this kind. Of course, with the low error rates obtained, they differ very little from the original correct-response distributions, but all have unit area. The first step, then, was to apply the correction to the three distributions of each subject separately.

For each of the three distributions, for each subject separately, we calculated 19 quantiles at .05 intervals from .05 to .95 . In addition, we constructed a fourth cumulative distribution that, at all times, consisted of the sums of the cumulative proportions for single presentation of the large and small targets. Summing to 2, the distribution would have 39 such quantiles, but we computed only the first 19 . Following Ratcliff (1979), we then obtained average distributions by computing the means of the subject quantiles at each .05 interval. These distributions are presented graphically in Figure 1. Smooth curves are drawn through the distributions for the global level (large target), local level (small target), and presentation at both levels. The curves were fitted by procedures described in the next section. Early quantiles for the distribution of sums are indicated by separate points only.

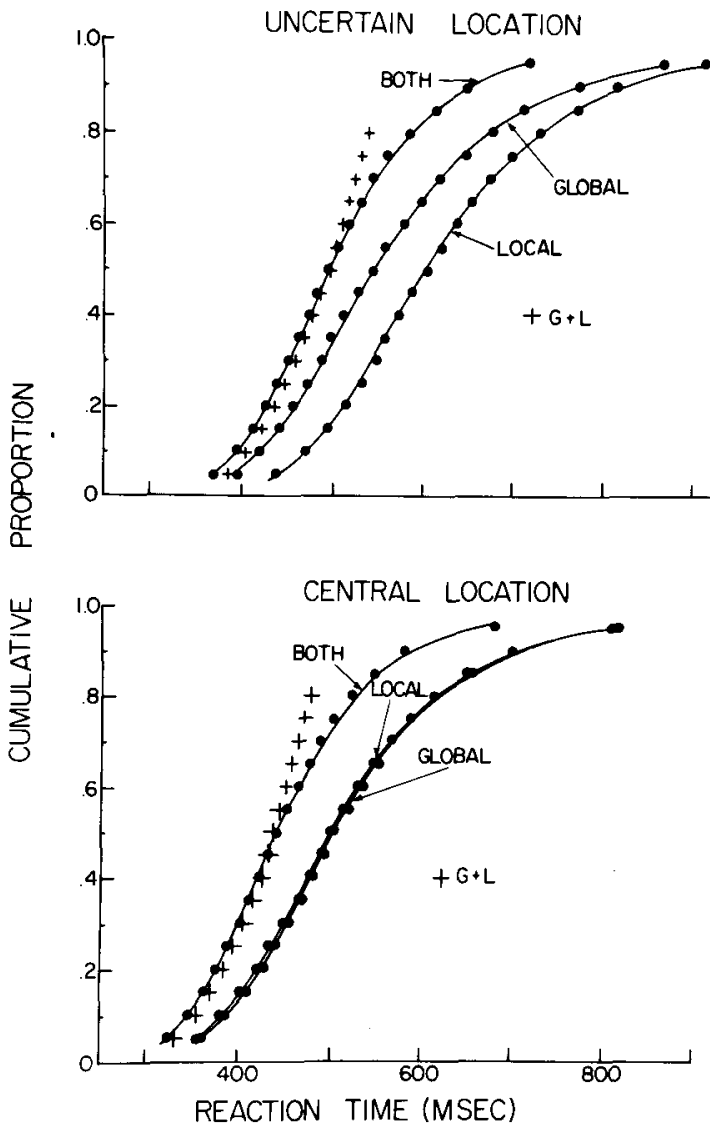

Figure 1. Obtained and calculated cumulative distributions for the three conditions in Experiment 1 (uncertain location) and Experiment 2 (central location). Separate points indicate the distributions of sums of the global and local conditions.

For Experiment 1, with uncertain stimulus location, the distributions for global and local stimuli are clearly separated, indicating the global precedence effect. In Experiment 2, these distributions are approximately superimposed, indicating the absence of the effect with central, fixed location. Also in Figure 1 , it may be observed that early quantiles for the distributions for presentation at both levels are consistently earlier than the corresponding quantiles for the distribution of sums. For Experiment 1, the distribution of sums does not equal and begin to exceed that for double stimulation until a proportion of .5 . For Experiment 2, this is at .4. Thus, Inequality 2, required by separate activation models, is consistently violated. At early quantiles, this violation is statistically significant. For Experiment 1 , the significant violations are as follows: $.05, \mathrm{t}(27)=3.945, \mathrm{p}<.001$; $.10, \mathrm{t}(27)=2.247, \mathrm{p}<.05 ; .15, \mathrm{t}(27)=2.175, \mathrm{p}<$ $.05 ; .20, \mathrm{t}(27)=2.012, \mathrm{p}<.05$, one tail; .25, $\mathrm{t}(27)=$ $1.704, \mathrm{p}<.05$, one tail. For Experiment 2, they are as follows: $t(27)=2.411, p<.05 ; .10, t(27)=1.854$, $\mathrm{p}<.05$, one tail; .20, $\mathrm{t}(27)=1.850, \mathrm{p}<.05$, one tail. 
The result of these experiments is that we have confirmed the conclusions of Miller (1981) that the class of separate activation models must be rejected. This does not mean that our basis for questioning his experimental design was incorrect. The differences between these quantiles that we have obtained, while significant, are considerably smaller than those he obtained.

Investigation of the combination rule. Since separate activation theory and statistical facilitation may be rejected for these data, the results suggest some form of summation principle in terms of strength. Thus our intent to examine this possibility gains added importance. Although we planned to consider some existing theories, we did not begin with a specific hypothesis concerning the summation rule, but intended to examine it empirically with the measurement methods of variable criterion theory.

Variable criterion theory has provided a useful approach in a number of contexts. Simply, it involves the notion that the tendency to respond to a stimulus gradually increases following the onset of that stimulus. When the tendency to respond reaches criterion strength, the response occurs. Variability is introduced into the theory by assuming that the criterion varies randomly from trial to trial. Systematic manipulation of the criterion affects the mean of the criterion distribution. When formulated more precisely, this simple model is useful in the analysis of data because it leads to scaling procedures that, in combination with experimental manipulation, make quantitative theoretical analysis possible.

The scaling procedures of variable criterion theory (e.g., Grice, 1972, Grice, Nullmeyer, \& Spiker, 1977, 1982), have been most recently summarized in the appendix by Grice, Canham, and Schafer (1982). In the RT context, excitatory strength (E) leading to response evocation is assumed to grow as a deterministic function of the time following stimulus onset. When $E$ reaches a criterion value specific to a trial, response evocation occurs. The criterion is assumed to be multiply determined and normally distributed over trials, with mean, $\bar{C}$, and standard deviation, $\sigma$. If $\bar{C}$ and $\sigma$ are taken as the origin and unit of the scale, then the cumulative probability of response $[P(t)]$ at time $t$ following stimulus onset is given by

$$
P(t)=\phi[E(t)],
$$

where $\phi$ is the integral of the unit normal function, and $E(t)$ is the value of the function for the growth of $E$ at $t$. The cumulative distribution, then, is determined by the growth function in combination with the normal distribution. ${ }^{1}$ This leads directly to a Thurstonean measurement model, by means of which the function for $\mathrm{E}$ may be estimated from empirical cumulative distributions. This model is as follows:

$$
\hat{E}(t)=\phi^{-1}[p(t)]=z(t),
$$

where $\hat{E}(t)$ is the estimate of $E(t)$, and $p(t)$ is the obtained cumulative proportion of responses at $t$. $\phi^{-1}$ is the inverse normal function or the normal deviate $[z(t)]$ of $p(t)$, with $p$ representing the lower tail of the normal distribution. In CRT with nonzero error rates, the appropriate distribution to which to apply the procedure is the cumulative distribution after correction by the race model.

In letter identification experiments not involving associative interference, the growth of $E$ has been interpreted as dependent on the growth of associative strength. The full relation is

$$
E(t)=[A(t)-\bar{C}] / o,
$$

where $A(t)$ is associative strength at $t$. As estimated directly from data, $\bar{C}=0$, and $\sigma=1$, so $E(t)=A(t)$. In all such experiments so far analyzed, associative strength has been found to grow as a simple, negatively accelerated, exponential growth function of time (Grice et al., 1983; Grice, Canham, \& Schafer, 1982). The function is of the form

$$
A(t)=a-m e^{-k t},
$$

where $a$ is the asymptote and $m$ and $k$ are constants. In the analysis of data, these constants are determined by curve-fitting procedures such that the resulting function precisely describes the data. At the present stage of theory, no attempt is made to assign theoretical meaning to the individual parameters.

Analyses of these experiments, in terms of strength, were performed with the same data as in the preceding analyses. Following Equation 4, the cumulative proportions at each of the 19 quantiles were transformed to normal deviates for the distributions of each experiment. When plotted as functions of time, these scales formed extremely smooth negatively accelerated functions in all cases. The functions for single targets at the global and local levels were then fitted with exponential growth functions of the form of Equation 6. For each of the four functions, the proportion of variance of the data points accounted for by the fitted equation was greater than .999 . Graphs of these equations are presented in Figure 2. In Experiment 1, the global precedence effect is clearly evident, in the different growth rates of global and local associative strength, while in Experiment 2 the functions are nearly identical.

The scale values obtained from Equation 4 include both positive and negative values. However, some of the possible summation models require all positive values. For example, the simple sum of two negative values will not account for a redundant-signals effect. For this reason, we have established a provisional origin at $\bar{C}-3.5$. This is the point at which the normal function rounds to zero in three decimal places, and not a rational absolute zero. For the following analysis, 3.5 has been added to all scale values. 


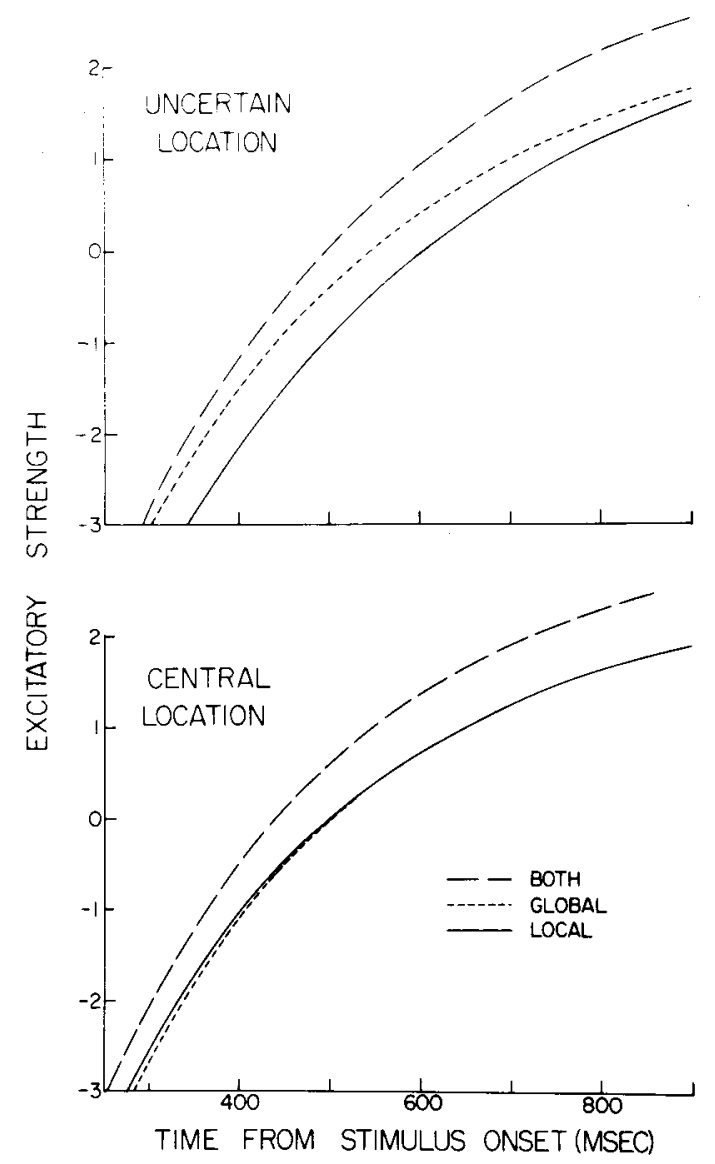

Figure 2. Calculated functions for the growth of excitatory strength for Experiment 1 (uncertain location) and Experiment 2 (central location).

The investigation of the combination rule began with the simplest possible summation principle-the relation between the function for targets at both levels and the sum of the two single-target conditions. Actually, this relation turned out to be so powerful that it effectively excluded other possibilities. In order to observe this relationship, we computed the values of the two single-target functions at the 19 quantiles of the redundant target conditions. In view of the goodness of fit, this is an extremely accurate way of representing the single-target data. The redundant target scale values were then plotted against the sums of two individual functions at the 19 quantiles. The result for each experiment was a precise linear relation. Plots of these relations are presented in Figure 3. The least squares regression equations describing the relations are as follows:

\section{Experiment 1,}

$$
\mathrm{E}_{\text {Both Levels }}=.524\left(\mathrm{E}_{\text {Global }}+\mathrm{E}_{\text {Local }}\right)+.572 \text {. }
$$

Experiment 2,

$$
\mathrm{E}_{\text {Both Levels }}=.508\left(\mathrm{E}_{\text {Global }}+\mathrm{E}_{\text {Local }}\right)+.551 \text {. }
$$

The value of $\mathbf{r}^{2}$ for Experiment 1 is greater than .999 . The value for Experiment 2 is $\mathbf{9 9 7}$. However, the slightly smaller value for Experiment 2 is a little misleading. If the discrepant, and least reliable, tail point at the .95 quantile is deleted, $r^{2}$ becomes .9995 for the remaining 18 points. The functions for both levels in Figure 2 were computed from Equations 7 and 8, and they accurately represent the data points with the precision of $r^{2}$. The smooth curves representing the distributions in Figure 1 were calculated by obtaining the normal function, Equation 3, of the functions in Figure 2. The proportion of variance accounted for in each of the six distributions is .999 or greater. This simple empirical model accounts for the data with remarkable accuracy.

These results indicate, throughout the distribution, a clear summation effect of the separate functions, each contributing about half of its strength. This summation is independent of the relative strengths of the two components. But, also throughout the distributions, there is an additional additive constant, represented by the intercept, that must be considered. An initial guess could be that this merely reflects the location of the origin. This turns out not to be true. For linear functions of this kind, the slope and the value of $r^{2}$ are unaffected by choice of the origin, that is, the size of an additive constant. The intercept is normally affected. For example, if the original scale values are used with $\bar{C}=0$, the intercept for $\mathrm{Ex}$ periment 1 is .742 rather than .572 and the intercept for Experiment 2 is .608 rather than .551. It may be shown that if the slope is exactly .5 the intercept does not change with changes in an additive constant. For

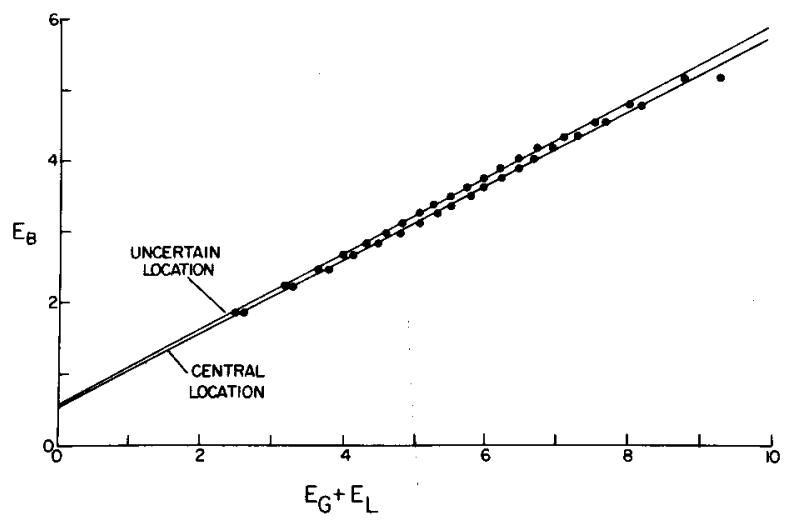

Figure 3. Relations between the strength of the redundant target conditions and the sum of the strengths of the separate target conditions for Experiment 1 (uncertain location) and Experiment 2 (central location). Points are at the 19.05 quantiles of the redundant target conditions. Lines are least squares regression lines. 
slopes greater than .5 , the intercept decreases with increases in the additive constant, but the change is very slow if the slope is near .5. For slopes less than .5 , the intercept increases with increases in the additive constant. For Experiment 1, it may be calculated that for the intercept to be zero the origin would be at $\bar{C}-15.5$. For Experiment 2, it would be at -76 . Both of these numbers are unrealistically low for a rational origin. For example, the range of the A functions do not include values as low as -15.5 for positive values of time. The basic conclusion, then, is that positive intercepts greater than zero are genuine. This, in turn, leads to a more substantive and rather startling conclusion. In spite of clear evidence of summation, it is not the summation effect that accounts for most of the redundant signals effect evident in the distribution for the combined stimuli. Rather, the effect reflects a constant increment, present throughout the entire distribution.

In spite of the precise relation obtained, we have examined three additional models that have been proposed, although none were proposed in the context of RT distributions. The earliest such theory was a rational equation presented by Hull (1939) to account for the combined effect of two stimuli associated with the same response. Developed in a different context, the equation does not apply directly to the present situation. What we have found is that, with a somewhat different interpretation and additional parameter estimation, the equation can be applied to our data. The equation involves summation according to a principle of decreasing gain. We have decided that further evaluation in a contemporary context should await experimental data to which the principle is more directly applicable. ${ }^{2}$

Fidell (1970), in the context of bimodal detection tasks, proposed a principle of vectorial summation of independent inputs. We found that this did not describe the present data. We also investigated the vectorial summation for correlated inputs. However, we failed to find relations required by such models for any acceptable origin of the scale. ${ }^{3}$

Kinchla and Collyer (1974) proposed a summation model, also with a Gaussian metric, in connection with letter detection when the number of redundant targets was a variable. The model was not applied to RT data. Their model suggested that the strength of combined targets was simply the weighted sum of the component strengths. Our results with the RT distributions differ in two respects. First, rather than full summation, we obtained only partial summation, as indicated by the slopes of the linear functions. Second, their model contains no additive constant as we found. ${ }^{4}$ The fact that our two components were equally weighted is not in conflict, since there were equal presentations of each stimulus. More recent data of Kinchla (1977) and Kinchla, Solis-Macias, and Hoffman (1983) strongly suggest that we should expect to find unequal weighting with conditions designed to bias attention with respect to the two levels.

\section{EXPERIMENTS 3 AND 4}

Experiments 3 and 4 investigated the redundantsignals effect with simple displays consisting of only two letters separated horizontally. The target was either letter or both. These CRT experiments were similar to ones conducted by Miller (1982) with the yes-no procedure. With two responses, right and left, these experiments involved an additional complication. Because of the S-R compatibility effect, one would expect faster responses when the target was on the same side as the required response. Thus, a demonstration of the redundant-signals effect requires that responses on redundant-target trials be faster than S-R compatible, single-target trials. Appropriate analyses are in terms of compatibility rather than simple position. The experiments are also of interest because the S-R compatibility effect has not previously been studied in terms of the growth of associative strength based on a scaling analysis. The two experiments differed only in the horizontal spacing between the two letters.

\section{Method}

Procedures in these experiments were identical to those of Experiments 1 and 2, except for the visual displays. The stimuli were the regular TRS-80 double-width capital letters as before. On each trial, two letters were presented equidistant horizontally from the fixation point. On one-third of the trials, the target was on the right, on one-third it was on the left, and on one-third it occupied both positions. The target letters were $H$ and $S$, and the irrelevant letter on single-target trials was $Y$. With this arrangement, the S-R compatible and incompatible trials were equally divided on single-target trials. The two mappings of the targets on the responses were equally divided among the subjects. In Experiment 3 the separation between the two letters was 6 deg, and in Experiment 4 the separation was .5 deg. There were 28 subjects in each experiment from the same population as above.

\section{Results}

Means of subject mean RTs and mean error rates are presented in Table 2. Reaction times were significantly faster with the close spacing of the letters in Experiment 4 than with the wide spacing of Experiment $3[F(1,54)=4.64, p<.05]$. The redundantsignals effect was evaluated by a comparison of the redundant-target condition with the single-target, compatible condition. The effect is significant $[F(1,54)$ $=49.21, \mathrm{p}<.001]$. The redundancy effect does not differ significantly between the two spacing experiments. For the redundancy $\times$ spacing interaction $[F(1,54)<1]$. The S-R compatibility effect is also significant in a comparison of the two single-target conditions $[F(1,54)=129.79, p<.001]$. The effect is significantly greater with the wide spacing of Experiment 3. The interaction, compatibility $\times$ spacing, is significant $[F(1,54)=13.38, \mathrm{p}<.001]$. It should be 
Table 2

Means and Standard Deviations of Mean Correct Reaction Time (in Milliseconds) and of Error Rates in Experiment 3 (Wide Spacing) and Experiment 4 (Close Spacing)

\begin{tabular}{|c|c|c|c|c|c|c|c|c|}
\hline \multirow{3}{*}{$\begin{array}{c}\text { Target } \\
\text { Condition }\end{array}$} & \multicolumn{4}{|c|}{ Reaction Time } & \multicolumn{4}{|c|}{ Error Rate } \\
\hline & \multicolumn{2}{|c|}{ Wide Spacing } & \multicolumn{2}{|c|}{ Close Spacing } & \multicolumn{2}{|c|}{ Wide Spacing } & \multicolumn{2}{|c|}{ Close Spacing } \\
\hline & Mean & SD & Mean & $\mathrm{SD}$ & Mean & SD & Mean & SD \\
\hline Both & 444 & 62 & 418 & 56 & .006 & .008 & .008 & .015 \\
\hline Compatible & 466 & 68 & 436 & 62 & .006 & .010 & .009 & .015 \\
\hline Incompatible & 511 & 74 & 459 & 57 & .022 & .018 & .030 & .031 \\
\hline
\end{tabular}

further noted that the compatibility effect is significant for Experiment 4 alone [ $\mathrm{t}(27)=6.685, \mathrm{p}<.001]$.

Although all error rates are low, in both experiments the error rate is significantly higher for the $\mathrm{S}-\mathrm{R}$ incompatible condition than for the other conditions. According to the sign test for all of these comparisons, $\mathrm{p}<.001$.

Tests of separate activation theory. In the same manner as above, we obtained the average cumulative distributions for the S-R compatible and incompatible conditions and for the redundant-target condition. Also, the distribution of sums of the two singletarget conditions was calculated. For the first four quantiles in Experiment 3, the quantiles for the twotarget conditions were slightly faster than those for the distribution of sums. For the .05 quantile, the difference was $11 \mathrm{msec}$ and significant $[\mathrm{t}(27)=2.418$, $\mathrm{p}<.05]$. Thus, the class of separate activation theories may be rejected for Experiment 3. Violations of Inequality 2 may occur at no point in the distributions according to such models. In Experiment 4, however, no such violations occurred with respect to the average quantiles. The differences were very small at the early quantiles, ranging from 3 to $9 \mathrm{msec}$ up to .25 quantile, but the quantiles for the distribution of sums were consistently faster. Separate activation models cannot be rejected on the basis of Miller's test. However, in view of the small early differences and the conservative nature of the test, the results provide no actual support for such theories.

For additional information, we inspected the distributions of all individual subjects, calculated at 20msec intervals. We found that 16 of the 28 subjects showed violations of Inequality 2 in one or more early class intervals. This frequency of violation is a real difficulty for separate activation theory. This is particularly true in view of Miller's (1982) finding that such violations are rare in simulations of such models.

In the absence of significance tests leading to the rejection of the class of separate activation models for Experiment 4, we have conducted tests of models of this type assuming independence. The tests are similar to ones recently used by Shaw (1982). According to such a model, the probability of not responding to the combined stimuli by time $t$ is equal to the product of the probabilities of not responding to the two stimuli separately; that is,

$$
p(t)_{A \& B}=p(t)_{A} p(t)_{B} \text {, }
$$

where the values of $p$ refer to the probability that a response has not occurred by $t$. Taking the logs of Equation 9 gives

$$
\ln p(t)_{A \& B}=\ln p(t)_{A}+\ln p(t)_{B}
$$

Equation 10 holds under independence and is used for the significance tests. For the distributions of each subject, we identified the class intervals and the probabilities where the cumulative proportions first equaled or exceeded .25 and .5 for the redundant target condition. The probabilities for the same class intervals were also obtained for the two single-target conditions, and the transformations of Equation 10 were applied. At both the .25 and .5 class intervals, Equation 10 significantly underpredicted the proportion of failures to respond. In other words, the cumulative proportions of responses were overpredicted [for the .25 interval, $t(27)=3.323, p<.01$; for the .5 interval, $\mathrm{t}(27)=5.499, \mathrm{p}<.001]$. Separate activation theory assuming independent inputs must be rejected on the basis of Experiment 4, as well as the other experiments.

From the point of view of separate activation theory, there is an interesting, but apparently unrecognized, possibility for the data from such experiments. Often it is possible to obtain quantitative, theoretical estimates of the correlation between the two channels. Using the data from the redundant- and single-target data in combination, it is possible to construct a fourfold table from which a phi coefficient or tetrachoric correlation may be computed. The proportions of responding and not responding to the separate targets provide estimates of the marginals of the table. The probability of not responding when both stimuli are present fills one cell of the table. Since the table has only one degree of freedom, the entire table is then determined. There are some empirical data that are not consistent with the construction of such a table. An instance is a violation of Inequality 2. From the average distributions of Experiment 4 , we have computed cosine- $\pi$ estimates of the tetrachoric correlation at the 19.05 quantiles of the redundant targets distribution. These correlations are all positive and fairly consistent throughout the dis- 
tribution. Their mean is .76 , and their standard deviation is .06 . The positive correlation is consistent with the direction of the discrepancies found in the application of Equation 10. It is worth noting that, for simulated data satisfying independence, correlations computed in this way do remain at zero, within rounding error, throughout the distribution.

Combination rule and S-R compatibility in terms of strength. The scaling procedures of variable criterion theory were applied to the average distributions in the same way as the preceding experiments. In both Experiment 3 and Experiment 4, the associative growth functions for the S-R compatible and incompatible conditions were precisely fitted with exponential functions. For all four of the functions, the proportion of variance accounted for was .999 . Graphs of the fitted equations are presented in the upper two panels of Figure 4. The dependence of the growth of associative strength on S-R compatibility is seen clearly. The target in the compatible position has an initial advantage from the earliest responses and also approaches its limit more rapidly. The two functions tend to converge at longer latency as the early advantage diminishes. The greater compatibility effect with the wide letter spacing of Experiment 3 is also clear in these functions. Since the trials occurred in an unpredictable order, the criterion parameters are, in principle, the same for the two functions. Thus, compatibility appears clearly as an associative phenomenon, as would be expected. These functions measure compatibility as a function of time, a form of analysis we have not previously seen.

For both experiments, the functions for the condition with both targets present were again precise linear functions of the sums of the single-target functions. These relations were again obtained at the 19 quantiles of the redundant-target conditions. No additive constant to change the origin was used in these analyses. The least squares regression equations were as follows:

\section{Experiment 3,}

$$
\begin{aligned}
& \mathrm{E}_{\text {Both Targets }} \\
& \quad=.489\left(\mathrm{E}_{\text {Compatible }}+\mathrm{E}_{\text {Incompatible }}\right)+.455 .
\end{aligned}
$$

Experiment 4,

$$
\begin{aligned}
& \mathrm{E}_{\text {Both Targets }} \\
& \quad=.500\left(\mathrm{E}_{\text {Compatible }}+\mathrm{E}_{\text {Incompatible }}\right)+.313 .
\end{aligned}
$$

In both instances, the value of $\mathbf{r}^{2}$ was .999. The functions for both targets in Figure 4 are computed from these equations and fit the empirical scale values with the precision of $r^{2}$. Fits to the cumulative RT distributions were obtained by taking the normal function of the calculated scale values at the quantiles of each distribution. For each of the six distributions, the proportion of variance accounted for was greater than .999.

In Experiment 3, the slope of the linear function is a little less than .5. This means that the intercept would increase slightly with increasing size of an additive constant used to shift the origin of the scale. In Experiment 4, with a slope of exactly .5, the intercept will not change with any additive constant. For these experiments, we may be certain that the redundant signals effect is not produced by the additive relation of the components alone, but that an additive constant is an essential component of the process.

\section{EXPERIMENT 5}

In Experiment 5, the stimuli also consisted of a simple two-letter display, but the letters were spaced vertically above and below the fixation point. The purpose of the experiment was to create a task in which the S-R compatibility effect would not be expected (Wallace, 1971). In this instance, the appropriate analysis of the redundant signals effect would be simply in terms of two spatial positions rather than in terms of compatibility.

\section{Method}

Except for the display, procedures in this experiment were the same as in the preceding experiments. The target letters and the irrelevant letter were the same. The two letters were presented equidistant from the fixation point directly above and below it. The vertical distance between the two letters was approximately $3 \mathrm{deg}$. The numbers of trials with the target in the upper position, lower position, and both positions were equal. There were $28 \mathrm{fe}$ male subjects from the same population as in the other experiments.

\section{Results}

As expected, there was no evidence of an S-R compatibility effect in this experiment. Mean RT for the two combinations of the right and left responses with targets in the upper and lower positions differed by less than $5 \mathrm{msec}$, and the difference was not significant $[\mathrm{t}(27)<1]$.

Analyzed in terms of position, the RT results in milliseconds were as follows: target in both positions, mean $=447$, $S D=53$; target in upper position, mean $=471, S D=59$; and target in lower position, mean $=503, \mathrm{SD}=64$. RTs were significantly faster when the target was in the upper rather than the lower position $[t(27)=7.024, p<.001]$. In a comparison of target in both positions with target in the upper position only, the redundant signals effect is significant $[t(27)=5.514, p<.001]$. The effect is about the same size as in the other experiments.

Error rates were as follows: both positions, mean = $.009, \mathrm{SD}=.012$; upper position, mean $=.016, \mathrm{SD}=$ .020 ; and lower position, mean $=.028, \mathrm{SD}=.025$. Errors for the lower position differed significantly 
from the other two. By the sign test, $p<.01$ for both comparisons.

Tests of separate activation theory. Data for Experiment 5 were analyzed in the same manner as the other experiments. In terms of the average quantiles, violations of Inequality 2 were minimal. The .05 quantile for both targets preceded that for the distribution of sums by just $3 \mathrm{msec}$. At the .10 quantile, there was a violation of $1 \mathrm{msec}$. These violations were not significant, and there were no others. The experiment provides no support for separate activation theory, but the class cannot be rejected on the basis of statistical significance. However, inspection of the individual distributions showed that 17 of the 28 subjects showed violations of Inequality 2 at one or more early intervals.

Evaluations of the independence model were performed as for Experiment 4. Independence predicts response proportion accurately or slightly underpredicts for the first four quantiles. After that, overpredictions of response proportions were similar to those of Experiment 4. The independence model was rejected by significant violations of Equation 10 at the .25 and .5 intervals [at $.25, t(27)=2.315, \mathrm{p}<.05$; at $.5, t(27)=3.274, p<.01]$. These were the only intervals tested, but overprediction continued to increase after the .5 interval.

Correlations were also computed where possible. The correlation was about zero at .2 , but after that became increasingly positive. It was .23 at $.25, .40$ at $.5, .52$ at .75 , and .71 at .9. Positive correlations imply a consistent overprediction of response proportions by the independence model.

Combination rule in terms of strength. The scaling analysis confirmed the pattern found in the other experiments. The associative functions for single targets in the upper and lower positions were precisely fitted with exponential growth functions. In both cases, the proportion of variance accounted for was .999. Graphs of the functions are presented in the bottom panel of Figure 4. The function for targets in both positions was again a linear function of the sums of the calculated values of the separate functions at the 19 quantiles. The linear regression equation is as follows:

$$
\mathrm{E}_{\text {Both Targets }}=.510\left(\mathrm{E}_{\text {Upper }}+\mathrm{E}_{\text {Lower }}\right)+.442 \text {. }
$$

The value of $r^{2}$ was .998. In this instance, eliminating a somewhat discrepant tail point at the .95 quantile increased the value of $r^{2}$ to .9996 for the remaining 18 points. ${ }^{5}$ With the slope and intercept of Equation 13, it may be shown that there will always be a positive intercept for any acceptable origin of the scale.

The function for both targets in Figure 4 is computed from Equation 13. The three cumulative RT distributions were fitted by taking the normal functions of the calculated functions at the quantiles. In

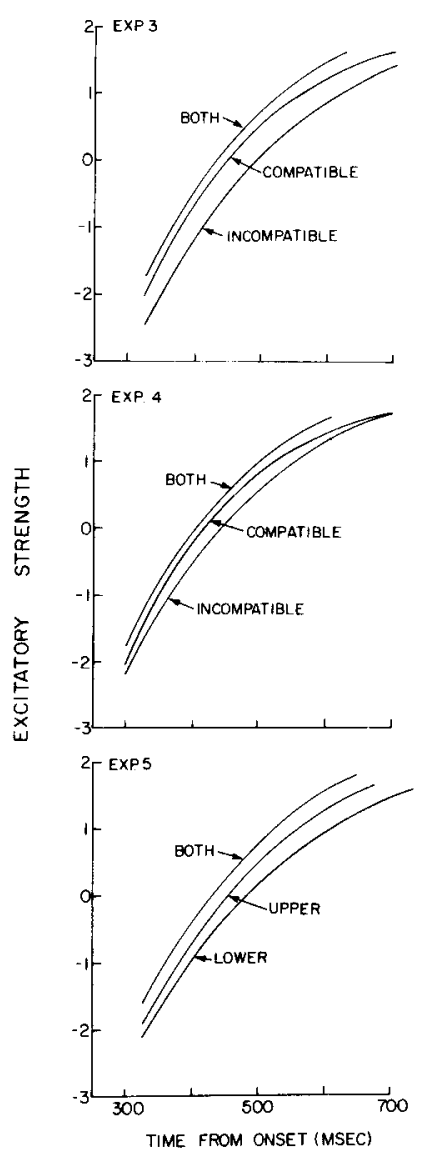

Figure 4. Calculated functions for the growth of excitatory strength for Experiment 3 (6-deg horizontal spacing), Experiment 4 (.5-deg horizontal spacing), and Experiment 5 (3-deg vertical spacing).

each instance, the proportion of variance in the data points accounted for is greater than .999 .

\section{DISCUSSION}

The evidence from these experiments is consistent and compelling in suggesting that the form of the RT distribution for redundant stimuli is determined by the sum of the strengths of the component stimuli. Throughout the distribution in this summation, each component contributes about one-half of its strength. This relation was obtained with quite different visual displays. Also, as indicated in Figures 3 and 4, the relation holds for substantial differences in the separation and forms of the two component functions. These facts indicate that the summation principle is a law of considerable generality.

It should be recognized that what we have here is not a formal deductive model. The linear, additive relations are theoretical statements in that they are based on the measurement model of variable criterion theory and purport to represent measurement of otherwise unobservable states of strength. However, 
their most immediate status is that of empirically observed relations between simple transformations of the experimentally obtained variable, response proportion, and time. Such simple and precise relations are seldom obtained in psychological research, and it is in this sense that they are so remarkable. The normal deviate transformation is dictated by the assumptions of variable criterion theory and was in no way adjusted to produce any particular form of combination rule. The credibility and utility of any measurement model is primarily based on the degree to which the results provide simple and orderly descriptions of empirical relations. These results provide strong support for variable criterion theory on this basis. The results appear to be too precise and too general to be the result of a fortuitous accident. Taken as a whole, the research presents nearly conclusive evidence that redundant stimuli combine on the basis of strength rather than as proportions following the rules of probability. In this respect, the results confirm the conclusions of Miller (1982) and provide evidence concerning the nature of the combination rule.

In spite of the evidence for an additive relationship, there remains an additional, extremely interesting question. This concerns the ubiquitous additive constant. Presumably, most theorists, including the authors, would have expected that, if an additive relation existed, it would account for the redundant signals effect. However, as pointed out earlier, this is not so. The matter was discussed earlier, but it may be put differently. If the slope were exactly .5 and there were no additive constant, the result would be a function composed of the means of the two component functions, and an additive constant would be required to account for the redundant-signals effect with any origin of the scale. In none of the present experiments was the slope sufficiently greater than .5 to account for the effect with any realistic origin. The question, then, is why is the additive constant present in combination with clear evidence for an additive effect? We suggest one possible explanation.

In research of this kind, theorists have generally assumed that measures of strength or response proportions obtained on single-target trials accurately estimate the contributions from each target that will be present on redundant-target trials. Our findings suggest that this assumption may be incorrect, and that the strength of each component may be a little greater on two-target trials. In other words, the strength of one component may be reduced if the other position or level is occupied by a distractor rather than by another target. This reduction might be called a distraction decrement, and our data indicate that it is a constant throughout the distribution. What is being summed, then, is not the separate strengths observed in the single-target conditions, but these strengths each incremented by the amount of the distraction decrement. The linear summation equations may be rewritten in the following manner for two stimuli, A and B:

$$
E_{A \& B}=s\left(E_{A}+d+E_{B}+d\right),
$$

where $d$ is the distraction decrement. The relation of this to the original form of the equation is given by

$$
\mathrm{d}=\mathrm{k} / 2 \mathrm{~s},
$$

where $\mathrm{k}$ is the intercept of the original form of the equation and $s$ is the slope. This form of the equation will hold for any origin of the scale, although $\mathrm{d}$ will change with the origin if the slope is not .5. This conceptualization at least makes the additive relation fully interpretable and $d$ becomes a measure of the distraction decrement. ${ }^{6}$ Potentially, $d$ could be used to measure the effect of different distractors, and the hypothesis in general seems open to experimental investigation in several directions.

The above interpretation does have some support from other research. Grice, Canham, and Schafer (1982) used a focused-attention task, similar to C. W. Eriksen and Schultz's (1979), in which a central target letter was flanked on each side by a noise letter. When the noise letter was identical to the target or an irrelevant letter, associative strength grew as an exponential growth function, as in the present experiments. The functions for identical and irrelevant noise letters were of the same shape, but that for irrelevant noise was a constant distance below the identical noise function throughout the entire distribution. The authors called this "perceptual interference"' to distinguish it from the associative interference obtained when the noise letter was associated with a competing response. This effect was consistent in five experiments. This appears to be the same phenomenon obtained here. Both involve the presence of an irrelevant letter, and both produce a decrement that is constant throughout the distribution. The effect was smaller in the earlier experiments than that indicated by the intercepts of the present summation functions. This makes sense. In the Grice, Canham, and Schafer (1982) experiments, the irrelevant letter was in an irrelevant position that the subject was instructed to ignore. In the present experiments, the irrelevant letter occupied a relevant location to which the subject was required to attend.

In addition to the above directly related data, there are various other sources of evidence indicating that the presence of irrelevant stimuli in a visual display retards response to a target. In summarizing some of these data, C. W. Eriksen and Schultz (1978) called this phenomenon "cognitive masking." More recently, Treisman, Kahneman, and Burkell (1983) and Kahneman, Treisman, and Burkell (1983) have interpreted such data as indicating "filtering cost." They attribute this cost to competition for attention. 
The above analysis suggests that the "redundant signals effect" in this situation is not produced by the additional information from a second target but by the absence of a distractor. Of course, it is possible that the presence of two stimuli adds a constant increment of associative strength to each throughout the distribution. Our chief reason for doubting this is that we should expect increments of associative strength to be cumulative rather than constant. However, this matter cannot be fully resolved without additional, related research.

The present experiments represent only one of the ways in which the redundant-signals effect has been studied. Most theorizing has been based on detection experiments in which the stimuli are presented in a brief exposure and the data are percentages of correct detections. This task is really quite different from that of RT experiments with long exposures and low error rates. We see no strong reason to believe that the same model should apply to both situations (Santee \& Egeth, 1982). Shaw (1982) has presented detection data in which she found an independence model to fit better than a simple Gaussian summation model in 24 of 34 comparisons made. Of course, a partial summation model with an additive constant, as found here, was not considered since it has never been proposed. In spite of the likelihood that different principles apply, we think it premature to reject out of hand the applicability of such a strength model to detection data. The application may be difficult, however, in the absence of that powerful analytic tool, the RT distribution.

There are two additional findings of interest which are supplementary to the main purpose of the research. The first concerns the global precedence effect. The results of Grice et al. (1983) have been confirmed in that global precedence is obtained with uncertain stimulus location, but not with a fixed, central location. The principle is also extended in that it is true for a divided-attention task as well as for a focused-attention task.

The other supplementary result concerns the S-R compatibility effect. In Experiments 3 and 4, the scaling analysis showed the time course of the effect in terms of the growth of associative strength. Also, the dependence of the effect on stimulus separation was shown in this way.

This is the first occasion on which the methods of variable criterion theory have been applied to average distributions obtained by Ratcliff's (1979) method. Frequently, analyses have been based on combined group distributions (Grice, Canham, \& Schafer, 1982, pp. 384-385). Here it was desirable to use the distributions based on mean quantiles so that tests of separate activation theory and the scaling analyses would be based on the same data. However, we have conducted additional analyses that show that the same conclusion would follow if the group distribution had been used in the scaling analyses.

\section{REFERENCES}

Duncan, J. (1980). The locus of interference in the perception of simultaneous stimuli. Psychological Review, 87, 272-300.

Eriksen, B. A., \& Eriksen, C. W. (1974). Effects of noise letters upon the identification of a target letter in a nonsearch task. Perception \& Psychophysics, 16, 143-149.

Eriksen, C. W., \& Schultz, D. W. (1978). Temporal factors in visual information processing: A tutorial review. In J. Requin (Ed.), Attention and Performance VII. Hillsdale, NJ: Erlbaum.

Eriksen, C. W., \& Schultz, D. W. (1979). Information processing in visual search: A continuous flow conception and experimental results. Perception \& Psychophysics, 25, 249-263.

Fidell, S. (1970). Sensory function in multimodel signal detection. Journal of the Acoustical Society of America, 47, 1009 . 1015.

Flowers, J. H., \& Wilcox, N. (1982). The effect of flanking context on visual classification: The joint contribution of interactions at different processing levels. Perception \& Psychophysics, 32, 581-591.

GrICE, G. R. (1972). Conditioning and a decision theory of response evocation. In G. H. Bower (Ed.), The psychology of learning and motivation (Vol. 5, pp. 1-65). New York: Academic Press.

Grice, G. R., Canham, L., \& Boroughs, J. M. (1983). Forest before trees? It depends where you look. Perception \& Psychophysics, 33, 121-128.

Grice, G. R., Canham, L., \& Schafer, C. (1982). Development of associative and perceptual interference. Perception \& Psychophysics, 32, 375-387.

Grice, G. R., Nullmeyer, R., \& Spiker, V. A. (1977). Application of variable criterion theory to choice reaction time. Perception \& Psychophysics, 22, 431-449.

Grice, G. R., Nullmeyer, R., \& Spiken, V. A. (1982). Human reaction time: Toward a general theory. Journal of Experimental Psychology: General, 111, 135-153.

Grice, G. R., \& SPIKer, V. A. (1979). Speed-accuracy tradeoff in choice reaction time: Within conditions, between conditions, and between subjects. Perception \& Psychophysics, 26, 118-126.

Grice, G. R., Spiker, V. A., \& Nullmeyer, R. (1979). Variable criterion analysis of individual differences and stimulus similarity in choice reaction time. Perception \& Psychophysics, 25, 353-370.

Hull, C. L. (1939). The problem of stimulus equivalence in behavior theory. Psychological Review, 25, 361-372.

Hull, C. L. (1943). Principles of behavior. New York: AppletonCentury.

Kahneman, D., Treisman, A., \& Burkell, J. (1983). The cost of visual filtering. Journal of Experimental Psychology: Human Perception and Performance, 9, 510-522.

Kinchu, , R. A. (1974). Detecting target elements in multielement arrays: A confusability model. Perception \& Psychophysics, 15, 149-158.

KINCHLA, R. A. (1977). The role of structural redundancy in the perception of visual targets. Perception \& Psychophysics, 22, 19-30.

Kinchla, R. A., \& Collyer, C. E. (1974). Detecting a target letter in briefly presented arrays: A confidence rating analysis in terms of a weighted additive effects model. Perception \& Psychophysics, 16, 117-122.

Kinchla, R. A., Solis-Macias, V., \& Hoffman, J. (1983). Attending to different levels of structure in a visual image. Perception \& Psychophysics, 33, 1-10.

Mrlle R, J. (1981). Global precedence in attention and decision. Journal of Experimental Psychology: Human Perception and Performance, 7, 1161-1174. 
Miller, J. (1982). Divided attention: Evidence for coactivation with redundant signals. Cognitive Psychology, 14, 247-279.

Navon, D. (1977). Forest before trees: The precedence of global features in visual perception. Cognitive Psychology, 9, 353-383.

RAAB, D. (1962). Statistical facilitation of simple reaction times. Transactions of the New York Academy of Sciences, 24, 574590.

RATCLIFF, R. (1979). Group reaction time distributions and an analysis of distribution statistics. Psychological Bulletin, 86, 446-461.

Santee, J. L., \& Egeth, H. E. (1982). Do reaction time and accuracy measure the same aspects of letter recognition? Journal of Experimental Psychology: Human Perception and Performance, 8, 489-501.

Shaw, M. L. (1982). Attending to multiple sources of information: 1. The integration of information in decision making. Cognitive Psychology, 14, 353-409.

Treisman, A., Kahneman, D., \& Bunkell, J. (1983). Perceptual objects and the cost of filtering. Perception \& Psychophysics, 33, 527-532.

Wallace, R. J. (1971). S-R compatibility and the idea of a response code. Journal of Experimental Psychology, 88, 354-360.

\section{NOTES}

1. Graphic illustrations of the relation between the normal distribution and a growth function in determining response proportions have been presented in several places, for example, Grice and Spiker (1979), Figure 5.

2. After its original presentation (Hull, 1939), Hull's equation was later presented in a number of other places. For two stimuli, $A$ and $B$, the equation is

$$
E_{A \& B}=E_{A}+E_{B}-\frac{E_{A} E_{B}}{E_{M A X}}
$$

$\mathrm{E}_{\mathrm{MAX}}$ is the limit of an exponential growth function describing the growth of associative strength as a function of the number of conditioning trials. The limit was assumed to be the same for each stimulus and for the combination. The combined strength is as if one stimulus had received the additional number of trials equal to those required to produce the strength of the other. If we wish to apply this logic to the passage of time, the assumption of equal limits is not true for the present data. However, we evaluated the possibility that there might be a single unknown value by calculating $E_{M A X}$ from the equation at each of the 19 quantiles of the combined target condition. $\mathrm{E}_{\mathrm{MAX}}$ was not constant, but increased as a monotonic function of time, so the original logic does not apply here to time.

We did observe that $\mathrm{E}_{\mathrm{MAX}}$ increased as a precise, linear function of $E_{A}+E_{B}$. Thus, by determining the linear parameters, it would be possible to fit the data by Hull's equation. However, in the absence of the original logic, this appears to have no advantage over the more direct additive relation. Hull (1943) also presented a more general equation for more than two stimuli. It is probably worth evaluating the principle of decreasing gain when the number of redundant targets is an experimental variable.

3. Fidell's (1970) model was in terms of $d$ ', which does have a rational origin. For two stimuli, $A$ and $B$, the relation for uncorrelated inputs is

$$
\mathrm{d}_{\mathrm{A \& B}}^{\prime}=\left(\mathrm{d}_{\mathrm{A}}^{\prime 2}+\mathrm{d}_{\mathrm{B}}^{\prime 2}\right)^{1 / 2} .
$$

For correlated inputs the relation is

$$
d_{A \& B}^{\prime 2}=d_{A}^{\prime 2}+d_{B}^{\prime 2}+2 d_{A}^{\prime} d_{B}^{\prime} \cos \theta,
$$

where $\cos \theta$ estimates the correlation. We explored these relations with values $E$ for origins within the range of possible values and were unable to obtain a fit.

4. Kinchla and Collyer (1974) obtained good fits of their model for two of their three observers. This was confirmed by a different and more recent analysis of their data by Shaw (1982). We have examined their data in a still different way. Computing values of $d^{\prime}$ from their data, we examined plots of $d^{\prime}$ as a function of the number of targets. The plots were linear for all three observers. For two of the observers, the intercepts were near zero, as predicted by their model. For the third observer, the intercept was well above zero, a result more like what we obtained with the RT distributions.

5. Methodologically, it is worth noting that, when average distributions are computed by Ratcliff's (1979) method, the reliability of the average quantiles tends to decrease monotonically with increasing quantiles. For Experiments 2 and 5, we have computed estimates of the standard error of the mean quantile at the .05 , $.5, .9$, and .95 quantiles. For Experiment 2, these estimates were 8.4, 12.2, 21.2, and $35.9 \mathrm{msec}$, respectively. For Experiment 5, the corresponding estimates were $6.1,9.2,17.6$, and $23.5 \mathrm{msec}$. The success of the present research in producing such consistent and orderly relationships obviously depended on the presence of very stable data. Apparently, the use of 80 trials per condition and 28 subjects per experiment provided such data.

6. It is not necessary to assume that $d$ is equal for the two stimuli, only that both are constant through the distribution. If they are not equal, then d from Equation 15 is the mean of the two values.

7. A description of the analysis relating the two kinds of average distributions is available from the authors. Equations for all of the fitted growth functions are also available.

(Manuscript received September 26, 1983; revision accepted for publication March 19, 1984.) 